\title{
INVARIANT OPERATORS AND UNIVALENT FUNCTIONS ${ }^{1}$
}

\author{
BY
}

\author{
REUVEN HARMELIN
}

\begin{abstract}
Necessary and sufficient conditions for univalence of meromorphic functions in certain domains in the complex plane are established in terms of some differential operators of degrees $\geqslant 3$, possessing the same invariance property as the Schwarzian derivative. Those operators include the derivatives of the Schwarzian derivative and Aharonov's invariants. Conditions for the existence of quasiconformal extensions are also achieved.
\end{abstract}

I. Introduction. Let $\mathscr{Q}(D)$ and $\Re(D)$ denote the linear spaces of all the analytic and meromorphic functions, respectively, at a domain $D$ in the complete complex plane $\hat{\mathbf{C}}=\mathbf{C} \cup\{\infty\}$, and let $\Re_{0}(D)$ be the set of all univalent elements in $\mathscr{R}(D)$. For every positive integer $n$ let

$$
B_{n}^{*}(D)=\left\{\phi \in \mathbb{Q}(D):\|\phi\|_{n, D}^{*}=\|\phi\|_{n}^{*}=\sup _{z \in D} d(z, \partial D)^{n}|\phi(z)|<\infty\right\}
$$

where $d(z, \partial D)$ is the euclidean distance of $z$ to the boundary $\partial D$ of $D$. If $D$ is a hyperbolic domain, with a hyperbolic density $\rho_{D}(z)=\rho(z)$, let

$$
B_{n}(D)=\left\{\phi \in \mathbb{Q}(D):\|\phi\|_{n, D}=\|\phi\|_{n}=\sup _{z \in D} \rho(z)^{-n}|\phi(z)|<\infty\right\} .
$$

(Here we consider the hyperbolic density $\rho_{D}(z)$ with constant curvature -4 , so that $\rho_{U}(z)=\left(1-|z|^{2}\right)^{-1}$ for the unit $\operatorname{disc} U$. $)$

The Schwarzian differential operator

$$
\Re(D) \ni f \mapsto\{f, z\}=\left[\left(\frac{f^{\prime \prime}}{f^{\prime}}\right),-\frac{1}{2}\left(\frac{f^{\prime \prime}}{f^{\prime}}\right)^{2}\right](z) \in \mathscr{M}(D)
$$

is a typical invariant operator in the sense that

$$
\{g \circ f, z\}=\{f, z\} \text { for every Möbius transformation } g \text {, }
$$

and it has the following two remarkable properties:

(i) If $f \in \mathscr{M}_{0}(D)$ and $\phi(z)=\{f, z\}$, then $\phi \in B_{2}^{*}(D)$ and

$$
\|\phi\|_{2, D}^{*} \leqslant 6
$$

Received by the editors December 5, 1980 and, in revised form, May 19, 1981. The contents of this paper have been presented to the American Mathematic Society at the 87th annual meeting, January 9, 1981.

1980 Mathematics Subject Classification. Primary 30C55, 30C60.

'This is a part of a Ph.D. Thesis, written at the Technion, Israel Institute of Technology, under the guidance of Professor Uri Srebro. My deepest thanks to Professor Uri Srebro for his useful remarks and valuable support. 
(Gehring [7]), and if $f$ has a $\mu$-quasiconformal extension into $\hat{\mathbf{C}} \backslash D$, then

$$
\|\phi\|_{2, D}^{*} \leqslant 6\|\mu\|_{\infty} .
$$

(ii) Conversely, if $\partial D$ is a finite disjoint union of points and quasicircles, and if $\phi \in B_{2}(D)$ with

$$
\|\phi\|_{2, D}<\varepsilon
$$

for some $\varepsilon=\varepsilon(\partial D)>0$, then $\phi(z)=\{f, z\}$ in $D$, for some quasiconformal automorphism $f$ of $\hat{\mathbf{C}}$ which is conformal in $D$ (Osgood [14]). In particular if $D$ is the unit disc $U$, then $\varepsilon(\partial U)=2$ (Nehari [13], Ahlfors-Weill [4]).

Here we show that there is a large class of differential operators $\tau: \mathfrak{R}(D) \rightarrow \mathfrak{N}(D)$ which are invariant in the sense that

$$
\tau(g \circ f)=\tau(f) \text { for every Möbius transformation } g
$$

and which possess properties similar to (i) and (ii), i.e., in terms of which we can formulate criteria for univalence and for quasiconformal extendability of meromorphic functions.

II. Homogeneous invariant operators-definitions. A differential operator $\tau$ in $\mathfrak{R}(D)$ is called invariant if it satisfies condition $\left(1.2^{\prime}\right)$. Let $\phi_{n}, n=2,3,4, \ldots$, denote the differential operators

$$
\phi_{n}(f, z)=\frac{1}{(n+1) !}\{f, z\}^{(n-2)}, \quad f \in \Re(D), z \in D .
$$

Evidently each $\phi_{n}$ is invariant (see Lavie [10]) and

$$
\begin{aligned}
& \phi_{2}(f, z)=\frac{1}{6}\{f, z\}, \\
& \phi_{n}(f, z)=\frac{1}{n+1} \phi_{n-1}^{\prime}(f, z)=\frac{3 !}{(n+1) !} \phi_{2}^{(n-2)}(f, z), \quad n \geqslant 3 .
\end{aligned}
$$

Another example of a sequence of invariant operators are the so-called Aharonov operators $\psi_{n}, n=2,3, \ldots$, which are defined in [1] by means of the generating function

$$
\begin{aligned}
F(f ; z, \zeta)=\frac{f^{\prime}(z)}{f(z)-f(\zeta)}-\frac{1}{z-\zeta}=\sum_{n=1}^{\infty} \psi_{n}(f, z)(\zeta-z)^{n-1}, \\
\quad z \in D,|\zeta-z|<d(z, \partial D) .
\end{aligned}
$$

From this definition Aharonov derived the recursion formula:

$$
\begin{aligned}
& \psi_{2}(f, z)=\frac{1}{6}\{f, z\}=\phi_{2}(f, z), \\
& \psi_{n}(f, z)=\frac{1}{n+1}\left[\psi_{n-1}^{\prime}(f, z)+\sum_{j=2}^{n-2} \psi_{j}(f, z) \psi_{n-j}(f, z)\right], \quad n \geqslant 3 .
\end{aligned}
$$

An operator $\tau_{n}$ in $\mathfrak{N}(D)$ is called homogeneous (of degree $n$ ) if it has the form

$$
\tau_{n}(f, z)=\sum_{m=1}^{[n / 2]} \sum_{k_{j} \geqslant 2, \Sigma_{j=1}^{m} k_{j}=n} a_{k_{1}, k_{2}, \ldots, k_{m}} \prod_{j=1}^{m} \phi_{k_{j}}(f, z), \quad n \geqslant 2,
$$


for some constants $a_{k_{1}, k_{2}, \ldots, k_{m}}, 1 \leqslant m \leqslant\left[\frac{n}{2}\right]$. A homogeneous operator $\tau_{n}$ is called normal if $a_{n}=1$. Note that every normal homogeneous operator $\tau_{n}$ of degree $n$ is an invariant differential operator of $\operatorname{order}(n+1)$. The operators $\phi_{n}, n=2,3,4, \ldots$, are the simplest example for normal homogeneous operators. On the other hand formula (2.2') implies, by induction, that each $\psi_{n}$ is also normal homogeneous.

III. Necessary conditions for univalence. In this section we prove a necessary condition for univalence and for quasiconformal extendability of $f \in \mathfrak{R}_{0}(D)$ in terms of every homogeneous operator $\tau_{n}$ : $\Re(D) \rightarrow \operatorname{Tl}(D)$. The proof is based on the following three lemmas:

LEMMA 1. If $f \in \Re_{0}(U)$, where $U$ is the unit disc, then

$$
\sum_{n=2}^{\infty}(n-1)\left|\psi_{n}(f, 0)\right|^{2} \leqslant 1
$$

and if $f$ has a $\mu$-quasiconformal extension into $\hat{\mathbf{C}} \backslash U$, then

$$
\sum_{n=2}^{\infty}(n-1)\left|\psi_{n}(f, 0)\right|^{2} \leqslant\|\mu\|_{\infty}^{2} \text {. }
$$

Proof. Aharonov proved (3.1) by showing that it is a new formulation of the classical area theorem. Similarly $\left(3.1^{\prime}\right)$ is just another form of Lehto's area theorem for univalent functions with quasiconformal extension [11].

Lemma 2. If $f \in \mathfrak{R}_{0}(D)$ for any domain $D$, then

$$
\sum_{n=2}^{\infty}(n-1) d(z, \partial D)^{2 n}\left|\psi_{n}(f, z)\right|^{2} \leqslant 1, \quad z \in D,
$$

and if $f$ has a $\mu$-quasiconformal extension into $\hat{\mathbf{C}} \backslash D$, then

$$
\sum_{n=2}^{\infty}(n-1) d(z, \partial D)^{2 n}\left|\psi_{n}(f, z)\right|^{2} \leqslant\|\mu\|_{\infty}^{2} .
$$

Proof. Fix any point $z \in D$ and set $h(\zeta)=f(r \zeta+z)$ where $r=d(z, \partial D)$. From (2.2) we conclude

$$
\psi_{n}(h, \zeta)=r^{n} \psi_{n}(f, r \zeta+z), \quad|\zeta|<1
$$

Hence

$$
\psi_{n}(h, 0)=r^{n} \psi_{n}(f, z) .
$$

Now, if $f \in \mathfrak{M}_{0}(D)$ then $h \in \mathfrak{K}_{0}(U)$, and if $f$ has a $\mu$-quasiconformal extension into $\hat{\mathbf{C}} \backslash D, h$ has a $\tilde{\mu}$-quasiconformal extension into $\hat{\mathbf{C}} \backslash U$, with $\|\tilde{\mu}\|_{\infty}=\|\mu\|_{\infty}$. The lemma follows by setting $h$ instead of $f$ in Lemma 1 and applying (3.3').

LEMMA 3. For every domain $D$, the differentiation operators $d^{l}: B_{n}^{*}(D) \ni \phi \mapsto \phi^{(l)}$ $\in B_{n+l}^{*}(D), l=0,1,2, \ldots, n \geqslant 1$, are bounded by constants $K(l, n)$ depending only on $l$ and $n$.

Proof. Let $\phi \in \mathbb{Q}(D)$ with

$$
|\phi(z)| \leqslant C d(z, \partial D)^{-n}, \quad C=\|\phi\|_{n}^{*}, z \in D, n \geqslant 1 .
$$


Fix a point $z \in D$ and let $R=d(z, \partial D), \Gamma_{r}=\{\zeta:|\zeta-z|=r\}$ for $0<r<R$. Cauchy's inequalities and (3.4) imply:

$$
\left|\phi^{(l)}(z)\right| \leqslant \frac{l !}{r^{l}} \max _{\zeta \in \Gamma_{r}}|\phi(\zeta)| \leqslant C l ! r^{-l} \max _{\zeta \in \Gamma_{r}} d(\zeta, \partial D)^{-n}=C l ! r^{-l}(R-r)^{-n}
$$

Hence

$$
\left|\phi^{(l)}(z)\right| \leqslant C l ! \min _{0<r<R} r^{-l}(R-r)^{-n}=C l !\left(\frac{n+l}{n}\right)^{n}\left(\frac{n+l}{l}\right)^{l} R^{-n-l} \text {. Q.E.D. }
$$

THEOREM 1. Every homogeneous operator $\tau_{n}$ has a positive constant $K^{*}\left(\tau_{n}\right)=K_{n}^{*}$, which depends only on $\tau_{n}$, such that

(i) if $f \in \mathfrak{T}_{0}(D)$, for any domain $D$, and $\phi=\tau_{n}(f, \cdot)$, then

$$
\|\phi\|_{n, D}^{*} \leqslant K_{n}^{*}
$$

(ii) if f has a $\mu$-quasiconformal extension into $\hat{\mathbf{C}} \backslash D$, then

$$
\|\phi\|_{n, D}^{*} \leqslant K_{n}^{*}\|\mu\|_{\infty},
$$

(iii)

$$
K^{*}\left(\psi_{n}\right) \leqslant 1 / \sqrt{n-1}, \quad n \geqslant 2
$$

Proof. For $\tau_{n}=\psi_{n}$ the theorem follows from Lemma 2. In particular $K^{*}\left(\psi_{2}\right)=1$ (cf. Gehring [7, Corollary 1, p. 563]). Next, Lemma 3 implies

$$
\left\|\phi_{n}(f)\right\|_{n}^{*}=\frac{3 !}{(n+1) !}\left\|\phi_{2}^{(n-2)}(f)\right\|_{n}^{*} \leqslant \frac{3 !}{(n+1) !} K(n-2,2)\left\|\phi_{2}(f)\right\|_{2}^{*} .
$$

Thus, $\left(3.5^{*}\right)$ and $\left(3.5^{* *}\right)$ for $\phi_{n}$ follow from the corresponding inequalities for $\phi_{2}=\psi_{2}$, i.e.

$$
K^{*}\left(\phi_{n}\right) \leqslant \frac{3 !}{(n+1) !} K(n-2,2) K^{*}\left(\phi_{2}\right)=\frac{3 !}{(n+1) !} K(n-2,2) .
$$

Finally, for the general homogeneous invariant operator $\tau_{n}\left(3.5^{*}\right)$ and $\left(3.5^{* *}\right)$ follow from (2.3) and the obvious inequality

$$
\left\|\prod_{j=1}^{m} \phi_{k_{j}}(f)\right\|_{n}^{*} \leqslant \prod_{j=1}^{m}\left\|\phi_{k_{j}}(f)\right\|_{k_{j}}^{*}, \quad \sum_{j=1}^{m} k_{j}=n . \quad \text { Q.E.D. }
$$

REMARK. Schwarz' lemma implies

$$
\rho_{D}(z) d(z, \partial D) \leqslant 1, \quad z \in D,
$$

for every hyperbolic domain $D$. Therefore it follows from $\left(1.1^{*}\right)$ and (1.1) that $B_{n}(D) \subseteq B_{n}^{*}(D)$. Conversely if $D$ is also simply-connected and $\infty \notin D$, then Koebe's $\frac{1}{4}$-theorem yields

$$
\rho_{D}(z) d(z, \partial D) \geqslant 1 / 4, \quad z \in D
$$


Therefore for such domains $B_{n}(D)=B_{n}^{*}(D)$ with equivalent norms. Hence, for such domains one can omit the asterisks in Theorem 1 and obtain

COROllaRY 1. If $D$ is a hyperbolic simply-connected domain in $\mathbf{C}$, then for every homogeneous operator $\tau_{n}$ and every $f \in \mathfrak{T}_{0}(D)$ the function $\phi(z)=\tau_{n}(f, z)$ satisfies

$$
\|\phi\|_{n, D} \leqslant K\left(\tau_{n}\right)
$$

where the constant $K\left(\tau_{n}\right)$ depends only on $\tau_{n}$. Furthermore, if $f$ has a $\mu$-quasiconformal extension into $\hat{\mathbf{C}} \backslash D$, then

$$
\|\phi\|_{n, D} \leqslant K\left(\tau_{n}\right)\|\mu\|_{\infty} .
$$

IV. Sufficient conditions. We now prove the following generalization of property (ii) (see Introduction) of the Schwarzian derivative for every normal homogeneous operator.

THEOREM 2. Let $C$ be either a circle in $\mathbf{C}$ or a quasicircle passing through $\infty$, let $D_{1}$ and $D_{2}$ be the components of $\hat{\mathbf{C}} \backslash C$ such that $\infty \notin D_{2}$ if $C$ is a circle. Then every normal homogeneous operator $\tau_{n}$ in $\Re\left(D_{2}\right)$ has a positive constant $\varepsilon_{n}=\varepsilon\left(C, \tau_{n}\right)$, depending only on $C$ and $\tau_{n}$, such that for any $\phi \in B_{n}\left(D_{2}\right)$ with norm $\|\phi\|_{n}<\varepsilon_{n}$ there is a quasiconformal automorphism $f$ of $\hat{\mathbf{C}}$ which is conformal in $D_{2}$ and $\tau_{n}(f, z)=\phi(z)$ in $D_{2}$.

Sketch of Proof. In order to prove this theorem one should find, for every small enough $\phi \in B_{n}\left(D_{2}\right)$, a univalent meromorphic solution $f(z)$ in $D_{2}$ for the differential equation $\tau_{n}(f, z)=\phi(z)$ of order $(n+1)$, and a quasiconformal extension of $f(z)$ into $D_{1}=\hat{\mathbf{C}} \backslash \bar{D}_{2}$. In other words one would like to have, near the origin in $B_{n}\left(D_{2}\right)$, a local cross-section for the following nonlinear mapping:

$$
\mathcal{T}_{c}^{(n)}: M\left(D_{1}\right) \ni \mu \rightarrow \tau_{n}^{\mu}=\tau_{n}\left(f_{\mu}\right) \in B_{n}\left(D_{2}\right)
$$

where $M\left(D_{1}\right)$ is the set of all Beltrami coefficients $\mu$ supported in $D_{1}$ (i.e., $M\left(D_{1}\right)$ is the open unit ball in the complex Banach space $L^{\infty}\left(D_{1}\right)$ of all the bounded-measurable complex-valued functions supported in $D_{1}$ ) and $f_{\mu}$ is the unique $\mu$-quasiconformal automorphism of $\hat{\mathbf{C}}$ which fixes the points $\{0,1, \infty\}$ (this normalization causes no loss of generality because of the invariance property of $\tau_{n}$ and since every $\mu$-quasiconformal automorphism of $\hat{\mathbf{C}}$ differs from $f_{\mu}$ by a left composition with a Möbius transformation). Ahlfors-Weill [4] gave such a local cross-section for $\tau_{2}=\phi_{2}$ and $D_{2}$ is the unit disc $U$. In the first part of our proof we use their result to give a cross-section for $\tau_{n}=\phi_{n}$ and $D_{2}=U$ (Lemma 4). In the second part of the proof we show that the mapping $\mathcal{T}_{c}^{(n)}$ of (4.1) is holomorphic, and we compute its derivative $D \sigma_{c}^{(n)}(0)$ at the origin (Lemma 5). Next we find a continuous global cross-section

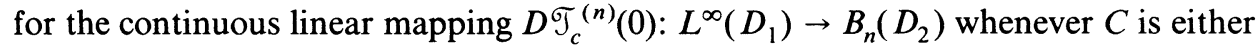
a quasicircle passing through $\infty$ (Lemma 6) or a circle (Lemma 6'). Thus the theorem is finally established by applying the inverse mapping theorem.

LEMMA 4. Every. $\phi \in B_{n+1}(U)$ has $a \psi \in B_{n}(U)$ such that $\psi^{\prime}=\phi$ and $\|\psi\|_{n} \leqslant$ $\left(2^{n} / n\right)\|\phi\|_{n+1}$. 
Proof. For every $\phi \in B_{n+1}(U)$ we simply define

$$
\psi(z)=\int_{0}^{z} \phi(\zeta) d \zeta=\int_{0}^{1} \phi(t z) z d t, \quad|z|<1 .
$$

Obviously $\psi^{\prime}(z)=\phi(z)$ in $U$ and

$$
\begin{aligned}
\left(1-|z|^{2}\right)^{n}|\psi(z)| & \leqslant \int_{0}^{1} \frac{\|\phi\|_{n+1}\left(1-|z|^{2}\right)^{n}}{(1-t|z|)^{n+1}}|z| d t \\
& =\frac{(1+|z|)^{n}}{n}\left[1-(1-|z|)^{n}\right]\|\phi\|_{n+1} \leqslant \frac{2^{n}}{n}\|\phi\|_{n+1}, \quad|z|<1,
\end{aligned}
$$

which proves the lemma.

Thus, if $\phi \in B_{n}(U)$ and

$$
\|\phi\|_{n} \leqslant \frac{1}{n(n+1)} 2^{1-(n+1)(n-2) / 2}
$$

then by successive use of Lemma 4 one can obtain a function $\psi \in B_{2}(U)$ such that $\phi(z)=\psi^{(n-2)}(z) /(n+1) !,|z|<1$, and

$$
\|\psi\|_{2} \leqslant \frac{2^{2+3+\cdots+(n-1)}}{(n-1) !}\left\|\psi^{(n-2)}\right\|_{n}<2 .
$$

Hence, by Ahlfors-Weill [4, Theorem A], $\psi(z)=\{f, z\}, z \in U$, where $f$ is a quasiconformal automorphism of $\hat{\mathbf{C}}$ which is conformal in $U$, and $\phi(z)=$ $\{f, z\}^{(n-2)} /(n+1) !=\phi_{n}(f, z)$. This completes the first part of the proof of Theorem 2 .

In the next lemma we show that infinitesimally all the mappings $\mathscr{T}_{c}^{(n)}$, defined in (4.1), behave the same as the mapping

$$
\Phi_{c}^{(n)}: M\left(D_{1}\right) \ni \mu \mapsto \phi_{n}^{\mu}=\phi_{n}\left(f_{\mu}, \cdot\right) \in B_{n}\left(D_{2}\right)
$$

near the center of $M\left(D_{1}\right)$.

LEMMA 5. The mapping $\mathscr{T}_{c}^{(n)}$, defined in (4.1), is a holomorphic function from $M\left(D_{1}\right)$ into $B_{n}\left(D_{2}\right)$, and its derivative at the origin is the continuous linear mapping

$$
\begin{array}{r}
D \mathscr{T}_{c}^{(n)}(0)=\mathfrak{M}_{c}^{(n)}: L^{\infty}\left(D_{1}\right) \ni \mu \mapsto-\frac{1}{\pi} \iint_{D_{1}} \frac{\mu(\zeta)}{(\zeta-z)^{n+2}} d \xi d \eta, \\
z \in D_{2}, \zeta=\xi+i \eta .
\end{array}
$$

Proof. Ahlfors and Bers [3] proved that $f_{t \mu}(z)$ is an analytic function of $t$ for every $z$ and $\mu \in M\left(D_{1}\right)$ when $t$ is small enough, and they showed that

$$
\left.\frac{\partial f_{t \mu}(z)}{\partial t}\right|_{t=0}=F_{\mu}(z)=-\frac{1}{\pi} \iint_{D_{1}}\left(\frac{1}{\zeta-z}-\frac{z}{\zeta-1}+\frac{z-1}{\zeta}\right) \mu(\zeta) d \xi d \eta,
$$


Later Bers proved in [6] that the mapping $\Phi_{c}^{(2)}: M\left(D_{1}\right) \rightarrow B_{2}\left(D_{2}\right)$ is holomorphic with the derivative

$$
\begin{aligned}
D \Phi_{c}^{(2)}(0): \mu & \left.\mapsto \frac{\partial \phi_{2}^{t \mu}(z)}{\partial t}\right|_{t=0}=\left(\mathfrak{M}_{c}^{(2)} \mu\right)(z)=\frac{1}{3 !} F_{\mu}^{(3)}(z) \\
& =-\frac{1}{\pi} \iint_{D_{1}} \frac{\mu(\zeta)}{(\zeta-z)^{4}} d \xi d \eta, \quad z \in D_{2} .
\end{aligned}
$$

But by $\left(2.1^{\prime}\right)$ we have

$$
\Phi_{c}^{(n)}=\frac{3 !}{(n+1) !} d^{(n-2)} \circ \Phi_{c}^{(2)} .
$$

Hence, by the chain rule, $\Phi_{c}^{(n)}$ is holomorphic and

$$
D \Phi_{c}^{(n)}(0)=\frac{3 !}{(n+1) !} d^{(n-2)} \circ D \Phi_{c}^{(2)}(0)
$$

and therefore (4.5) implies

$$
\begin{aligned}
D \Phi_{c}^{(n)}(0) & =\frac{3 !}{(n+1) !} d^{n-2} \circ \mathfrak{M}_{c}^{(2)}: \mu \mapsto\left(\mathfrak{M}_{c}^{(n)} \mu\right)(z) \\
& =\frac{1}{(n+1) !} F_{\mu}^{(n+1)}(z)=-\frac{1}{\pi} \iint_{D_{1}} \frac{\mu(\zeta)}{(\zeta-z)^{n+2}} d \xi d \eta
\end{aligned}
$$

Now, notice that the following product of holomorphic mappings

$$
\prod_{j=1}^{m} \Phi_{k_{j}}: M\left(D_{1}\right) \rightarrow B_{n}\left(D_{2}\right), \quad \sum_{j=1}^{m} k_{j}=n
$$

is holomorphic, and therefore $\widetilde{T}_{c}^{(n)}$ is holomorphic. Hence

$$
D \mathscr{T}_{c}^{(n)}(0)[\mu]=\lim _{t \rightarrow 0} \frac{1}{t}\left(\mathscr{T}_{c}^{(n)}(t \mu)-\mathcal{T}_{c}^{(n)}(0)\right)=\lim _{t \rightarrow 0} \frac{\tau_{n}^{t \mu}-\tau_{n}^{0}}{t}=\left.\frac{\partial \tau_{n}^{t \mu}}{\partial t}\right|_{t=0}
$$

and since $\phi_{k_{j}}^{0}(z)=\phi_{k_{j}}(\mathrm{id}, z) \equiv 0$, Leibnitz rule and (2.3) yield

$$
D \mathcal{G}_{c}^{(n)}(0)[\mu]=\left.\frac{\partial \tau_{n}^{t \mu}}{\partial t}\right|_{t=0}=\left.\frac{\partial \phi_{n}^{t \mu}}{\partial t}\right|_{t=0}=D \Phi_{c}^{(n)}(0)[\mu]=\mathfrak{M}_{c}^{(n)} \mu .
$$

This implies (4.3). Finally, for every $z \in D_{2}$ we get

$$
\begin{aligned}
\left|\left(\mathfrak{M}_{c}^{(n)} \mu\right)(z)\right| & \leqslant \frac{\|\mu\|_{\infty}}{\pi} \iint_{D_{1}|\zeta-z|^{n+2}} \frac{d \xi d \eta}{\mid \zeta \|_{\infty}} \iint_{|\zeta-z|>d(z, C)}|\zeta-z|^{-n-2} d \xi d \eta \\
& \leqslant \frac{\|}{\pi} \\
& =\frac{2}{n} d\left(z, \partial D_{1}\right)^{-n}\|\mu\|_{\infty}, \quad \mu \in L^{\infty}\left(D_{1}\right) .
\end{aligned}
$$

Hence, by (3.7) we conclude that $\mathfrak{M}_{c}^{(n)}: L^{\infty}\left(D_{1}\right) \rightarrow B_{n}\left(D_{2}\right)$ is bounded, and this completes the proof of Lemma 5. 
Ahlfors proved in [2, Lemma 2] that every quasicircle $C$ passing through $\infty$ admits a quasiconformal reflection $h$ (i.e. an involution $h: \mathbf{C} \rightarrow \mathbf{C}$ such that $\left.h\right|_{c}=$ id and $z \rightarrow \overline{h(z)}$ is quasiconformal) which is uniformly bilipschizian in $\mathbf{C}$, i.e.:

$$
K^{-1}\left|z_{1}-z_{2}\right| \leqslant\left|h\left(z_{1}\right)-h\left(z_{2}\right)\right| \leqslant K\left|z_{1}-z_{2}\right|
$$

for some constant $K=K(C)>1$ and all $z_{1}, z_{2} \in \mathbf{C}$. For $z_{2} \in C$ we have in particular

$$
\left|z_{1}-z_{2}\right| \leqslant K\left|h\left(z_{1}\right)-z_{2}\right|
$$

LEMMA 6. Let $C$ be a quasicircle passing through $\infty$, and $h$ a bilipschizian quasiconformal reflection at $C$. Then the linear mapping

$$
\Lambda_{c}^{(n)}: B_{n}\left(D_{2}\right) \ni \psi \mapsto(n+1)(\zeta-h(\zeta))^{n} \frac{\partial h(\zeta)}{\partial \bar{\zeta}} \psi(h(\zeta)) \in L^{\infty}\left(D_{1}\right),
$$

$$
\zeta \in D_{1}
$$

is a continuous right-inverse of $\mathfrak{M}_{c}^{(n)}: L^{\infty}\left(D_{1}\right) \rightarrow B_{n}\left(D_{2}\right)$.

Proof. We will show that the mapping $\Lambda_{c}^{(n)}$ is indeed from $B_{n}\left(D_{2}\right)$ into $L^{\infty}\left(D_{1}\right)$, is bounded and satisfies the reproduction formula

$$
\begin{aligned}
\psi(z)=\left(\mathfrak{M}_{c}^{(n)} \circ \Lambda_{c}^{(n)} \psi\right)(z)=-\frac{1}{\pi} \iint_{D_{1}} \frac{\left(\Lambda_{c}^{(n)} \psi\right)(\zeta)}{(\zeta-z)^{n+2}} d \xi d \eta, & \\
& z \in D_{2}, \psi \in B_{n}\left(D_{2}\right) .
\end{aligned}
$$

Observe, first, that according to formulas (4.8) and (4.4)

$$
\begin{aligned}
& \left(\mathfrak{M}_{c}^{(n)} \mu\right)(z)=\frac{F_{\mu}^{(n+1)}(z)}{(n+1) !} \\
& \quad=\frac{1}{(n+1) !} \frac{d^{n+1}}{d z^{n+1}}\left\{-\frac{z(z-1)}{\pi} \iint_{D_{1}} \frac{\mu(\zeta) d \xi d \eta}{(\zeta-z) \zeta(\zeta-1)}\right\}, \quad z \in D_{2},
\end{aligned}
$$

where $F_{\mu}(z)$ is known [8, pp. 129, 136] as the unique continuous solution in $C$ of the nonhomogeneous Cauchy-Riemann equation

$$
\partial F(z) / \partial \bar{z}=\mu(z)
$$

which satisfies $F_{\mu}(0)=F_{\mu}(1)=0$ and

$$
F_{\mu}(z)=O(|z| \log |z|) \quad \text { as } z \rightarrow \infty .
$$

But also if $\tilde{F}_{\mu}(z)$ is any other continuous solution of (4.13) which satisfies only

$$
\tilde{F}_{\mu}(z)=O\left(|z|^{2}\right) \quad \text { as } z \rightarrow \infty
$$

then by Weyl's lemma and Liouville's theorem $\tilde{F}_{\mu}-F_{\mu}$ is a polynomial of degree $\leqslant 2$, and hence

$$
\left(\mathfrak{M}_{c}^{(n)} \mu\right)(z)=\frac{\tilde{F}_{\mu}^{(n+1)}(z)}{(n+1) !}, \quad z \in D_{2}, n \geqslant 2
$$


Thus, in order to reproduce a Beltrami differential $\mu=\Lambda_{c}^{(n)} \psi \in L^{\infty}\left(D_{1}\right)$ with $\mathfrak{M}_{c}^{(n)} \mu=\psi$ for every given $\psi \in B_{n}\left(D_{2}\right)$, one should, first, choose an analytic solution $F(z)$ in $D_{2}$ of the equation

$$
F^{(n+1)}(z)=(n+1) ! \psi(z), \quad z \in D_{2} .
$$

Next one has to find an extension of this $F(z)$ into $D_{1}=\hat{\mathbf{C}} \backslash \overline{D_{2}}$ which would have a generalized derivative $\mu=\partial F / \partial \bar{z} \in L^{\infty}\left(D_{1}\right)$ with a $L^{\infty}$-norm comparable to $\|\psi\|_{n, D_{2}}$.

We assume, first, that $\psi$ belongs to the following subspace of $B_{n}\left(D_{2}\right)$ :

$\tilde{B}_{n}\left(D_{2}\right)=\left\{\psi \in B_{n}\left(D_{2}\right): \psi=\left.\hat{\psi}\right|_{D_{2}}\right.$ where $\hat{\psi} \in B_{n}\left(D_{0}\right)$ for some $\left.D_{0}=D_{0}(\psi) \supset \bar{D}_{2}\right\}$.

Then the solution $F(z)$ of $(4.15)$, for $\psi \in \tilde{B}_{n}\left(D_{2}\right)$, has a continuous extension to $\bar{D}_{2}=D_{2} \cup C$. Moreover, we have

$$
|\psi(z)|=|\hat{\psi}(z)| \leqslant\|\hat{\psi}\|_{n, D_{0}} \cdot \rho_{D_{0}}(z)^{n}=O\left(|z|^{-2 n}\right) \quad \text { as } z \rightarrow \infty \text { from } D_{2},
$$

since $\infty \in C \subset D_{0}$ and $\rho_{D_{0}}(z)=O\left(|z|^{-2}\right)$ as $z \rightarrow \infty$. Hence we can choose a solution $F(z)$ of $(4.15)$ such that

$$
F(z)=O\left(|z|^{-2 n+n+1}\right)=O\left(|z|^{2}\right) \quad \text { as } z \rightarrow \infty .
$$

Now, following Bers' method in [6] we extend $F(z)$ as follows:

$$
\hat{F}(z)= \begin{cases}F(z), & z \in D_{2} \cup C, \\ \sum_{k=0}^{n} \frac{(z-h(z))^{k}}{k !} F^{(k)}(h(z)), & z \in D_{1} .\end{cases}
$$

Obviously $\hat{F}(z)$ is well defined and continuous in C. Moreover, by the chain rule, (4.9) and (4.15), the generalized derivative $\partial \hat{F} / \partial \bar{z}$ exists almost everywhere and we have

$$
\frac{\partial \hat{F}}{\partial \bar{z}}=\frac{(z-h(z))^{n}}{n !} \frac{\partial h(z)}{\partial \bar{z}} F^{(n+1)}(h(z))=\left(\Lambda_{c}^{(n)} \psi\right)(z), \quad z \in D_{1} .
$$

Now we show that $\mu=\Lambda_{c}^{(n)} \psi \in L^{\infty}\left(D_{1}\right)$ for every $\psi \in B_{n}\left(D_{2}\right)$. Indeed, by (4.9') we have for every $z \in D_{1}$ and $z_{0} \in C$ :

$$
|z-h(z)| \leqslant\left|z-z_{0}\right|+\left|h(z)-z_{0}\right| \leqslant(K+1)\left|h(z)-z_{0}\right| \text {. }
$$

Hence, by (3.6) $|z-h(z)| \leqslant(K+1) d(h(z), C) \leqslant(K+1) \rho_{D_{2}}(h(z))^{-1}$. Observe also that (4.9) implies that the generalized derivative $\partial h(z) / \partial \bar{z}$ is essentially bounded by $K$, and therefore

$$
\begin{aligned}
\left|\left(\Lambda_{c}^{(n)} \psi\right)(z)\right| & \leqslant(n+1)(K+1)^{n} K \rho_{D_{2}}(h(z))^{-n}|\psi(h(z))| \\
& \leqslant(n+1)(K+1)^{n} K\|\psi\|_{n, D_{2}}, \quad z \in D_{1},
\end{aligned}
$$

which proves also the continuity of the linear mapping $\Lambda_{c}^{(n)}: B_{n}\left(D_{2}\right) \rightarrow L^{\infty}\left(D_{1}\right)$. Finally, the above construction yields

$$
\begin{aligned}
\left(\mathfrak{M}_{c}^{(n)} \circ \Lambda_{c}^{(n)} \psi\right)(z)=\left(\mathfrak{M}_{c}^{(n)} \mu\right)(z)=\frac{\hat{F}^{(n+1)}(z)}{(n+1) !}=\frac{F^{(n+1)}(z)}{(n+1) !} & =\psi(z), \\
& \quad z \in D_{2}, \psi \in \tilde{B}_{n}\left(D_{2}\right),
\end{aligned}
$$


i.e., every $\psi \in \tilde{B}_{n}\left(D_{2}\right)$ satisfies the required reproduction formula

$$
\psi(z)=-\frac{n+1}{\pi} \iint_{D_{1}} \frac{(\zeta-h(\zeta))^{n}}{(\zeta-z)^{n+2}} \frac{\partial h(\zeta)}{\partial \bar{\zeta}} \psi(h(\zeta)) d \xi d \eta, \quad z \in D_{2} .
$$

Suppose now that $\psi \in B_{n}\left(D_{2}\right)$. Then by [6, Lemma 1] there is a sequence $\left\{\psi_{j}\right\}$ in $\tilde{B}_{n}\left(D_{2}\right)$ which pointwise converges to $\psi$ in $D_{2}$, and such that $\left\|\psi_{j}\right\|_{n} \leqslant\|\psi\|_{n}$, $j=1,2, \ldots$. Then $\left\{\Lambda_{c}^{(n)} \psi_{j}\right\}_{j=1}^{\infty}$ converges to $\Lambda_{c}^{(n)} \psi$ pointwise in $D_{1}$, and is bounded in $L^{\infty}\left(D_{1}\right)$, since $\Lambda_{c}^{(n)}: B_{n}\left(D_{2}\right) \rightarrow L^{\infty}\left(D_{1}\right)$ is bounded. Hence, Lebesgue's bounded convergence theorem yields

$$
\begin{aligned}
\psi_{j}(z) & =-\frac{1}{\pi} \iint_{D_{1}} \frac{\left(\Lambda_{c}^{(n)} \psi_{j}\right)(\zeta)}{(\zeta-z)^{n+2}} d \xi d \eta \\
\downarrow & \downarrow \\
\psi(z) & =-\frac{1}{\pi} \iint_{D_{1}} \frac{\left(\Lambda_{c}^{(n)} \psi\right)(\zeta)}{(\zeta-z)^{n+2}} d \xi d \eta
\end{aligned} \quad\left(z \in D_{2}\right)
$$

which completes the proof of Lemma 6.

Lemma 6 implies the following analogue of Lemma 4:

LEMMA 4'. Let $C$ be a quasicircle passing through $\infty$, and $l \geqslant n \geqslant 2$. Then there is a constant $K=K(C, l, n)$, depending only on $C$, l and $n$, such that for every $\psi \in B_{l}\left(D_{2}\right)$ there is a function $\phi \in B_{n}\left(D_{2}\right)$ with $\phi^{(l-n)}=\psi$ and $\|\phi\|_{n} \leqslant K\|\psi\|_{l}$.

Proof. Formula (4.8) implies

$$
\mathfrak{M}_{c}^{(l)}=\frac{(n+1) !}{(l+1) !} d^{l-n} \circ \mathfrak{M}_{c}^{(n)}, \quad l \geqslant n \geqslant 2,
$$

and since $\mathfrak{M}_{c}^{(l)} \circ \Lambda_{c}^{(l)}=\mathrm{id}_{B_{l}\left(D_{2}\right)}$, it follows that the continuous linear mapping $((n+1) ! /(l+1) !) \mathfrak{M}_{c}^{(n)} \circ \Lambda_{c}^{(l)}: B_{l}\left(D_{2}\right) \rightarrow B_{n}\left(D_{2}\right)$ is a right inverse of the differentiation mapping $d^{l-n}: B_{n}\left(D_{2}\right) \rightarrow B_{l}\left(D_{2}\right)$, which proves the lemma.

LeMmA 6 . Let $D_{2}$ be a disc in C. For every integer $n \geqslant 2$ the mapping $\mathfrak{M}_{c}^{(n)}$ : $L^{\infty}\left(D_{1}\right) \rightarrow B_{n}\left(D_{2}\right)$ has a continuous cross-section. In particular, for $D_{2}=U$ and $n=2$, the linear mapping

$$
B_{2}(U) \ni \psi \mapsto\left(\Lambda_{c}^{(2)} \psi\right)(\zeta)=-3 \frac{\left(|\zeta|^{2}-1\right)^{2}}{\bar{\zeta}^{4}} \psi\left(\frac{1}{\bar{\zeta}}\right) \in L^{\infty}(\hat{\mathbf{C}} \backslash U),
$$

$|\zeta|>1$

is a continuous right-inverse of the mapping $\mathfrak{M}_{c}^{(2)}: L^{\infty}(\hat{\mathbf{C}} \backslash U) \rightarrow B_{2}(U)$.

Proof. It is enough to prove the lemma for the unit disc $U$. Ahlfors-Weill proved in [4] that the bounded linear mapping

$$
\left\{\phi \in B_{2}(U):\|\phi\|_{2}<2\right\} \ni \phi \mapsto \frac{1}{6}\left(\Lambda_{c}^{(2)} \phi\right)(\zeta) \in M(\hat{\mathbf{C}} \backslash U), \quad|\zeta|>1,
$$

is a local cross-section of the holomorphic mapping $M(\hat{\mathbf{C}} \backslash U) \ni \mu \mapsto 6 \phi_{2}^{\mu}=\left\{f_{\mu}, \cdot\right\}$ $\in B_{2}(U)$, i.e. $\Phi_{c}^{(2)} \circ \Lambda_{c}^{(2)}=$ id in the ball of radius 2 around the origin in $B_{2}(U)$. 
Hence, by the chain rule and (4.5)

$$
D \Phi_{c}^{(2)}(0) \circ \Lambda_{c}^{(2)}=\mathfrak{M}_{c}^{(2)} \circ \Lambda_{c}^{(2)}=\mathrm{id}_{B_{2}(U)} .
$$

This with Lemma 4 proves the lemma.

We now return to the proof of Theorem 2. By Lemma 5 the mapping $\mathscr{T}_{c}^{(n)}$ : $M\left(D_{1}\right) \rightarrow B_{n}\left(D_{2}\right)$ is holomorphic with the derivative $\mathfrak{M}_{c}^{(n)}: L^{\infty}\left(D_{1}\right) \rightarrow B_{n}\left(D_{2}\right)$ at the origin. By Lemmas 6 and $6^{\prime} \mathfrak{M}_{c}^{(n)}$ has a continuous cross-section $\Lambda_{c}^{(n)}: B_{n}\left(D_{2}\right) \rightarrow$ $L^{\infty}\left(D_{1}\right)$. Hence, the restriction of $\mathfrak{M}_{c}^{(n)}$ to the closed subspace $\operatorname{Im} \Lambda_{c}^{(n)}$ of $L^{\infty}\left(D_{1}\right)$ is a topo-isomorphism of $\operatorname{Im} \Lambda_{c}^{(n)}$ onto $B_{n}\left(D_{2}\right)$. Therefore, by the inverse mapping theorem there are neighborhoods $\Re_{1}$ and $\Re_{2}$ of the origins in $M\left(D_{1}\right) \cap \operatorname{Im} \Lambda_{c}^{(n)}$ and in $B_{n}\left(D_{2}\right)$, respectively, such that $\left.\mathcal{T}_{c}^{(n)}\right|_{\mathscr{T}_{1}}$ is a holomorphic homeomorphism of $\mathfrak{T}_{1}$ onto $\Re_{2}$, and in particular $\Re_{2} \subseteq \operatorname{Im} \mathscr{T}_{c}{ }^{(n)}$, i.e. $\operatorname{Im}_{\mathcal{T}_{c}}{ }^{(n)}$ in $B_{n}\left(D_{2}\right)$ contains an open ball of a positive radius $\varepsilon_{n}$ around the origin. This completes the proof of the theorem.

\section{REFERENCES}

1. D. Aharonov, A necessary and sufficient condition for univalence of a meromorphic function, Duke Math. J. 36 (1969), 599-604.

2. L. V. Ahlfors, Quasiconformal reflections, Acta Math. 109 (1963), 291-301.

3. L. V. Ahlfors and L. Bers, Riemann's mapping theorem for variable metrics, Ann. of Math. 72 (1960), 385-404.

4. L. V. Ahlfors and G. Weill, A uniqueness theorem for Beltrami equation, Proc. Amer. Math. Soc. 13 (1962), 975-978.

5. A. F. Beardon and F. W. Gehring, Schwarzian derivative, Poincaré metrics and the kernel function, Comment. Math. Helv. 55 (1980), 50-64.

6. L. Bers, A non-standard integral equation with applications to quasiconformal mappings, Acta Math. 116 (1966), 113-134.

7. F. W. Gehring, Univalent functions and the Schwarzian derivative, Comment. Math. Helv. 52 (1977), $561-572$.

8. I. Kra, Automorphic forms and Kleinian groups, Benjamin, New York, 1972.

9. W. Kraus, Über den Zusammengang einiger Charakteristiken eines einfach zusammenhängenden Bereiches mit der Kreis abbildung, Mitt. Math. Sem. Giessen 21 (1932), 1-28.

10. M. Lavie, The Schwarzian derivative and disconjugacy of $n$-th order linear differential equations, Canad. J. Math. 21 (1969), 235-249.

11. O. Lehto, Schlicht functions with a quasiconformal extension, Ann. Acad. Sci. Fenn. Ser. A I Math. 500 (1971), 1-10.

12. , Conformal mappings and Teichmüller spaces, Lectures at the Technion, Haifa, Israel, April-May 1973.

13. Z. Nehari, Schwarzian derivatives and schlicht functions, Bull. Amer. Math. Soc. 55 (1949), 545-551.

14. B. Osgood, Univalence criteria in multiply connected domains, Trans. Amer. Math. Soc. 260 (1980), 459-473.

Department of Mathematics, University of Maryland, College Park, Maryland 20742

Current address: Department of Mathematics, University of Michigan, Ann Arbor, Michigan 48109 